\section{Kidney \\ Blood Pressure Research}

\title{
The Impact of Umbilical Blood Flow Regulation on Fetal Development Differs in Diabetic and Non-Diabetic Pregnancy
}

\author{
Jian Lia You-Peng Chen ${ }^{b} \quad$ Yun-Peng Dong ${ }^{c} \quad$ Cai-Hong Yu ${ }^{a} \quad$ Yong-Ping Lu ${ }^{b}$ \\ Xiao-Min Xiaod Berthold Hochere,f
}

\begin{abstract}
aDepartment of Clinical Medicine, Medical college of Hunan Normal University, Changsha 410006; 'Department of Infectious Diseases, the First Affiliated Hospital of Jinan University, Guangzhou 510630; 'Department of Otolaryngology \& Hearing Research Laboratory, Second Xiangya Hospital, Central South University, Changsha 410011; 'Department of Obstetrics and Gynecology, the First Affliated Hospital of Jinan University, Guangzhou 510630, China; eInstitute of Nutritional Science, University of Potsdam, D-14558 Nuthetal-Potsdam; ' Center for Cardiovascular Research/Institute of Pharmacology, 10115 Berlin, Germany
\end{abstract}

\section{Key Words}

Umbilical artery Doppler - Blood flow resistance - Gestational diabetes mellitus • Fetal development

\begin{abstract}
Background/Aims: Diabetes is well-known to influence endothelial function. Endothelial function and blood flow regulation might be different in diabetic and non-diabetic pregnancy. However, the impact of umbilical blood flow regulation in gestational diabetes on fetal development is unknown so far. Methods: In a prospective birth cohort study, we analyzed the association of the umbilical artery Doppler indices (pulsatility index, resistance index and systolic/diastolic ratio) and fetal size measures (biparietal diameter, head circumference, abdominal circumference, femur length and birth weight) in 519 non-gestational diabetes mellitus pregnancies (controls) and 226 gestational diabetes mellitus pregnancies in middle (day $160.32 \pm 16.29$ of gestation) and late (day $268.12 \pm 13.04$ of gestation) pregnancy. Results: Multiple regression analysis considering confounding factors (gestational day of ultrasound examination, offspring sex, maternal body mess index before pregnancy, maternal age at delivery, maternal body weight at delivery and maternal hypertension) showed that umbilical artery Doppler indices (pulsatility index, resistance index and systolic/diastolic ratio) were associated with fetal head circumference and femur length in middle gestational diabetes mellitus pregnancy but not in non-gestational diabetes mellitus pregnancy. Head circumference, biparietal diameter, abdominal circumference and femur length in mid gestation were smaller in fetus of gestational diabetes mellitus pregnancy versus nongestational diabetes mellitus pregnancy. In contrast to non-gestational diabetes mellitus

Jian Li, PhD \&

Prof. Berthold Hocher, MD, PhD

Department of Clinical Medicine, Medical college of Hunan Normal University, Changsha 410006 (China), E-Mail 13656355@qq.com \&

Institute of Nutritional Science, University of Potsdam, 14558 Nuthetal, Potsdam

(Germany), E-Mail hocher@uni-potsdam.de, Homepage: http://www.uni-potsdam.de/eem
\end{abstract}




\section{Kidney Blood Pressure Research}

Kidney Blood Press Res 2014;39:369-377

DOI: 10.1159/000355815

(C) 2014 S. Karger AG, Basel

www.karger.com/kbr

Li/Chen/Dong/Yu/Lu/Xiao/Hocher: Umbilical Blood Flow Regulation and Fetal Development

pregnancy in late gestation, umbilical artery Doppler indices in gestational diabetes mellitus pregnancy were not associated with ultrasound measures of fetal growth. Birth weight was slightly increased in gestational diabetes mellitus pregnancy as compared to non-gestational diabetes mellitus pregnancy. Conclusions: The impact of umbilical blood flow on fetal growth is time dependent in human gestational diabetes mellitus and non-gestational diabetes mellitus pregnancy. In gestational diabetes mellitus pregnancy umbilical blood flow is critical for organ development in much earlier stages of pregnancy as compared to non-gestational diabetes mellitus pregnancy. The physiological and molecular pathways why there is a catch up growth in later times of gestational diabetes mellitus pregnancy resulting in larger gestational diabetes mellitus babies at birth needs to be addressed in further studies.

Copyright (c) 2014 S. Karger AG, Basel

\section{Introduction}

The prevalence of type 2 diabetes is rising at an alarming rate especially in Asia. A recent report by the International Diabetes Foundation projected that, by 2030, up to 438 million people may be affected by type 2 diabetes, accounting for more than $4.5 \%$ of the world's projected population [1]. In China alone, a recent survey reported that 92.4 million adults have diabetes [2]. Nowadays more and more younger people are affected such as pregnant women. This is particularly important, since diabetes may not just affect the health of the pregnant lady, but also affect substantially the health of the next generation. The prevalence of GDM in different countries varies depending on ethnicity and diagnosis standard [3-5]. Recent evidence also suggested that the prevalence of GDM itself is rising, possibly in parallel with the increasing prevalence of type 2 diabetes [6]. GDM is recognized as a risk factor for a number of adverse outcomes during pregnancy, including pre-eclampsia, cesarean section, fetal macrosomia, increased incidence of birth trauma due to macrosomia and shoulder dystocia, and neonatal metabolic abnormalities such as neonatal hypoglycemia. Maternal GDM is further more associated with long-term adverse consequences in the offspring and subsequent generations, in particular GDM is an important driver of the escalating global burden of diabetes and cardiovascular disease $[7,8]$. The fetus gets oxygen and nutrition via the umbilical vessels, thus blood flow in the vessel is essential for fetal development. Diabetes is well-known to influence endothelial function $[9,10]$. Thus, endothelial function and blood flow regulation might be different in diabetic and non-diabetic ladies. We therefore analysed the relationship of umbilical blood flow regulation and fetal growth in GDM and non-GDM pregnancy using modern 3D ultrasound technologies in a huge Asian birth cohort.

\section{Materials and Method}

\section{Clinic data collection}

The study was approved by the ethics committee of Jinan University, Guangzhou, China. We obtained written informed consent from all participants involved in our study. We invited a total of 932 Chinese women who delivered their babies at the obstetric department of the first affiliated hospital of Jinan university between March 2010 to January 2011 to participate in the study. Inclusion criteria were as follows: (1) the newborn was born without congenital anomalies; (2) singleton pregnancy; (3) no HIV and no syphilis. (4) oral glucose tolerance testing (OGTT) was performed in 2nd trimester of pregnancy. After exclusion of cases that did not fulfill the inclusion criteria or were not willing to participate, we finally included 745 remaining cases. After obtaining written consent, a structured medical history was taken. Chinese guidelines for medical follow-up in pregnancy comprise a perinatal health manual which contains essential data about the pregnancy. The data in the 'Perinatal health manual' were also used to judge whether the women fulfilled all inclusion and exclusion criteria. The following data were extracted into our database: Pregnancy woman: nationality/ethnic background, age, body height, body weight before and during pregnancy, gravidity, parity, gestational age at delivery, smoking before/during pregnancy, and alcohol consumption during pregnancy and blood pressure readings at all follow-up visits. Infant baby: birth weight, infant gender, gestational day 


\section{Kidney \\ Blood Pressure Research}

Kidney Blood Press Res 2014;39:369-377

\begin{tabular}{l|l}
\hline DOI: $10.1159 / 000355815$ & C) 2014 S. Karger AG, Basel \\
Publisned ontIne: september 30, 2014 & www.karger.com/kbr
\end{tabular}

Li/Chen/Dong/Yu/Lu/Xiao/Hocher: Umbilical Blood Flow Regulation and Fetal Development

at delivery, Apgar score at 1, 5, 10 min. Gestational age was calculated from the first day of the last normal menstrual period and confirmed by either first or early second trimester ultrasound scans. Biometric data of the newborns were routinely measured immediately after delivery.

The criteria for diagnosis of pregnancy-induced hypertension were based on the definition of the Chinese hypertension society: systolic blood pressure of $140 \mathrm{~mm} \mathrm{Hg}$ and/or a diastolic blood pressure of 90 $\mathrm{mm} \mathrm{Hg}$ after 20 weeks gestation, measured at least at two occasions with a break of 4 to 6 hours in-between. The criteria for diagnosis of gestational diabetes (GDM): plasma glucose concentrations were measured at 0 , 60 , and 120 minutes when the woman received at $75 \mathrm{~g}$ OGTT in the second trimester of pregnancy. GDM was diagnosed when patient plasma glucose levels exceeded or reached one of the following thresholds: fasting glucose $\geq 5.6 \mathrm{mmol} / \mathrm{L}$; one hour glucose $\geq 10.3 \mathrm{mmol} / \mathrm{L}$ or two hour glucose $\geq 8.6 \mathrm{mmol} / \mathrm{L}$.

\section{Ultrasound measurements during pregnancy}

Ultrasound measurements during pregnancy were done as described previously [11]. All the anthropometric parameters of the newborns were measured in two types of three-dimensional ultrasound examination units with an integrated image analysis software (MEDISON ACCUVIX V20, South Korea and GE VOLUSON 730 EXPERT, USA). Only obstetric physicians that passed the examination for ultrasound diagnostics during pregnancy performed the analysis ensuring the quality of the data. The biparietal diameter (BPD) was measured from the outer edge of the parietal bone near the probe to the inner edge of the other side of the parietal bone at the cross-sectional plane of the fetal brain with major land marks including the cavum septi pellucidi, thalamus, third ventricle and ambient cistern. The head circumference (HC) was measured in the same plane as the biparietal diameter using the elliptic function of the ultrasound instrument. Abdominal circumference (AC) was measured in a plane at the level of the fetal umbilical plexus perpendicular to the spine including the spine, stomach bubble, liver, umbilical vein, the skin and subcutaneous fat. Femur length (FL) was measured using predefined anatomic points that can easily be detected by ultrasound techniques in a standard manner (from the greater trochanter to the lateral condyle).

Doppler ultrasonography was performed with an ultrasound system with a 2.5- to 6-MHz curvilinear transducer (MEDISON ACCUVIX V20, South Korea and GE VOLUSON 730 EXPERT, USA). Color Doppler imaging was used to optimize the insonation by the pulsed Doppler examination. The angle of insonation was kept at $<15$ degrees in all cases and angle correction was used if the angle was not zero. The highpass filter was set at minimum, and a large sample volume $(10-12 \mathrm{~mm})$ was used for the pulsed Doppler recording. The Doppler velocity waveforms were obtained from the free-floating loop of the umbilical cord during fetal quiescence. Five to 6 uniform waveforms were obtained $>3$ times in succession, and online measurements were performed. The values that were recorded were an average of 3 consecutive cardiac cycles. The waveform envelope that had the highest measured peak systolic velocity was considered for analysis, assuming that the highest measured velocity represents the lowest angle of insonation. The guidelines of the International Perinatal Doppler Society were followed during Doppler sonographic examinations. The mechanical index was kept at $<1.9$, and the thermal index was kept at $<1.5$.Three Doppler waveform indices (pulsatility index(PI), resistance index(RI) and systolic/diastolic ratio(S:D ratio)) were calculated from the maximum velocity waveform with the following computerized planimetry:

$\mathrm{PI}=($ Peak systolic velocity/end diastolic velocity $) /$ time averaged maximum velocity

$\mathrm{RI}=($ Peak systolic velocity/end diastolic velocity $) /$ peak systolic velocity

$\mathrm{S}: \mathrm{D}$ ratio $=$ Peak systolic velocity/end diastolic velocity

\section{Data analysis}

Data were analyzed with SPSS version 19.0. Results are presented as mean \pm standard deviation. Bivariate correlation analyses was used to estimate the relationship between fetal size (BPD, HC, AC, FL, birth weight) and the umbilical artery (UA) Doppler indices (PI, RI and S/D ratio) in middle and late pregnancy, then the correlation was further analyzed by multivariable regression analysis considering gestational day of the ultrasound examination, gestational day at delivery, the child's sex, maternal body mess index (BMI) before pregnancy, maternal age at delivery, maternal body weight at delivery and maternal hypertension disorder state. These confounding variables showed a significant bivariate correlation or association with birth weight or ultrasound parameters in our cohort. Pearson's correlation coefficient and the regression coefficient B were used to estimate the strength of a correlation. A p-value of less than 0.05 was considered significant. 


\section{Kidney Blood Pressure Research}

\section{Kidney Blood Press Res 2014;39:369-377}

DOI: 10.1159/000355815

Publisned onIIne: september 30, 2014

C 2014 S. Karger AG, Base

www.karger.com/kbr

Li/Chen/Dong/Yu/Lu/Xiao/Hocher: Umbilical Blood Flow Regulation and Fetal Development

Table 1. Detailed descriptive data of the mothers and newborns

\begin{tabular}{|c|c|c|c|}
\hline Variable & Non-GDM (n=519) & $\operatorname{GDM}(n=226)$ & $P$ \\
\hline \multicolumn{4}{|l|}{ Mothers } \\
\hline Maternal age, years & $28.32 \pm 3.52$ & $29.48 \pm 3.54$ & $0.00^{\bullet}$ \\
\hline Gravidity & $1.69 \pm 1.06$ & $1.68 \pm 1.00$ & 0.94 \\
\hline Parity & $1.15 \pm 0.41$ & $1.16 \pm 0.45$ & 0.88 \\
\hline Maternal height,cm & $160.06 \pm 4.49$ & $159.52 \pm 4.83$ & 0.14 \\
\hline BMI before pregnancy, $\mathrm{kg} / \mathrm{m}^{2}$ & $20.11 \pm 2.33$ & $20.64 \pm 2.46$ & $0.01^{\bullet}$ \\
\hline Maternal weight before pregnancy, $\mathrm{kg}$ & $51.58 \pm 6.79$ & $52.57 \pm 7.13$ & 0.07 \\
\hline Maternal weight at delivery, kg & $67.76 \pm 7.93$ & $68.16 \pm 8.58$ & 0.53 \\
\hline Pregnancy-induced hypertension & 15 & 13 & 0.10 \\
\hline Preeclampsia & 12 & 10 & 0.17 \\
\hline \multicolumn{4}{|l|}{ Newborns } \\
\hline Child birth weight, $g$ & $3266.52 \pm 430.25$ & $3301.06 \pm 408.30$ & 0.31 \\
\hline Gestational age at delivery, day & $274.42 \pm 9.69$ & $274.70 \pm 8.03$ & 0.70 \\
\hline Child sex , male / female , \% & $52.6 \pm 47.4$ & $55.3 \pm 44.7$ & \\
\hline Apgar score at $1 \mathrm{~min}$ & $8.92 \pm 0.54$ & $8.88 \pm 0.46$ & 0.24 \\
\hline Apgar score at $5 \mathrm{~min}$ & $9.95 \pm 0.27$ & $9.94 \pm 0.29$ & 0.47 \\
\hline Apgar score at $10 \mathrm{~min}$ & $9.99 \pm 0.13$ & $9.98 \pm 0.13$ & 0.69 \\
\hline Late ultrasound analysis time, day & $268.12 \pm 13.04$ & $267.35 \pm 14.17$ & 0.47 \\
\hline Late BPD, mm & $94.45 \pm 4.69$ & $94.05 \pm 4.50$ & 0.28 \\
\hline Late $\mathrm{HC}, \mathrm{mm}$ & $330.56 \pm 13.92$ & $329.84 \pm 12.70$ & 0.51 \\
\hline Late $\mathrm{AC}, \mathrm{mm}$ & $338.26 \pm 22.59$ & $337.51 \pm 22.83$ & 0.68 \\
\hline Late $\mathrm{FL}, \mathrm{mm}$ & $70.71 \pm 3.94$ & $70.75 \pm 3.83$ & 0.91 \\
\hline Late UA-Doppler indices analysis time, day & $237.64 \pm 24.89$ & $235.33 \pm 22.10$ & 0.24 \\
\hline Late UA-S/D ratio & $2.55 \pm 0.51$ & $2.65 \pm 0.56$ & $0.03^{\bullet}$ \\
\hline Late UA-RI & $0.60 \pm 0.10$ & $0.62 \pm 0.11$ & 0.07 \\
\hline Late UA-PI & $0.90 \pm 0.19$ & $0.97 \pm 0.25$ & $0.00 \bullet$ \\
\hline Middle ultrasound analysis time, day & $160.32 \pm 16.29$ & $157.18 \pm 14.54$ & $0.02 \bullet$ \\
\hline Middle BPD, mm & $56.62 \pm 7.28$ & $55.20 \pm 6.73$ & $0.02^{\bullet}$ \\
\hline Middle HC, mm & $204.2725 \pm 27.27$ & $201.0224 \pm 24.47$ & 0.14 \\
\hline Middle AC, mm & $180.94 \pm 25.72$ & $175.39 \pm 24.75$ & $0.01^{\star}$ \\
\hline Middle FL, mm & $39.06475 \pm 6.02$ & $37.70 \pm 5.72$ & $0.01^{\star}$ \\
\hline Middle UA-S/D ratio & $3.50 \pm 0.76$ & $3.52 \pm 0.75$ & 0.74 \\
\hline Middle UA-RI & $0.71 \pm 0.09$ & $0.72 \pm 0.12$ & 0.17 \\
\hline Middle UA-PI & $1.18 \pm 0.21$ & $1.17 \pm 0.21$ & 0.53 \\
\hline
\end{tabular}

\section{Result}

Description of the cohort

745 pregnant women participated in the study. The OGTT in 2nd trimester of pregnancy revealed that 226 pregnant ladies had GDM according to the diagnostic criteria (see method section) and 519 pregnant women did not have GDM. Among of the 226 GDM cases, 134 had only one glucose value in the OGTT above the threshold, the remaining 92 cases had glucose levels above the threshold of at least 2 times in the OGTT. This indicates that the majority of pregnancy related GDM cases were mild in our study cohort.

Descriptive data of non-GDM and GDM pregnancy were provided in table 1. The following data between non-GDM and GDM group were significantly different (maternal age at delivery, $28.32 \pm 3.52$ years vs $29.48 \pm 3.54$ years. maternal BMI before pregnancy, $20.11 \pm 2.33 \mathrm{~kg} / \mathrm{m}^{2} \mathrm{vs}$ $20.64 \pm 2.46 \mathrm{~kg} / \mathrm{m}^{2}$. middle BPD, $56.62 \pm 7.28 \mathrm{~cm}$ vs $55.20 \pm 6.73 \mathrm{~cm}$. middle AC, $180.94 \pm 25.72$ $\mathrm{cm}$ vs $175.39 \pm 24.75 \mathrm{~cm}$. middle FL, $70.71 \pm 3.94 \mathrm{~cm}$ vs $37.70 \pm 5.72 \mathrm{~cm})$. Umbilical blood flow parameters such as UA-S/D ratio and UA-PI were different between GDM and non-GDM in late pregnancy stages, but not in middle pregnancy. 15 cases had pregnancy-induced hypertension in non-GDM group and 13 cases in GDM group; 12 cases had preeclampsia in non-GDM group and 10cases in GDM group, both of them were not significantly different between the 


\section{Kidney Blood Pressure Research}

two group. (for more details see table 1 ).

Fetal size with UA-Doppler indices by bivariate correlation analyses

Bivariate correlation analyses showed that parameters describing the fetus in late gestation (birth weight, $\mathrm{BPD}, \mathrm{HC}, \mathrm{AC}$, FL) were negative related to the late Doppler indices (PI, $\mathrm{RI}$ and S/D ratio) in non-GDM group. However, in the GDM group, just the parameters describing the fetus in middle gestation (BPD, HC, $\mathrm{AC}, \mathrm{FL})$ were negative related to the middle Doppler indices (PI, RI, and $S / D$ ratio) indicating basic differences in the relationship bet-
Table 2. Correlation Matrix of fetal size with UA-Doppler indices

\begin{tabular}{|c|c|c|c|c|c|}
\hline \multicolumn{6}{|c|}{ in non-GDM group } \\
\hline In middle pregnancy & & Middle BPD & Middle HC & Middle AC & Middle FL \\
\hline Middle UA-PI & & -0.31 & -0.33 & -0.33 & -0.32 \\
\hline Middle UA-RI & & -0.27 & -0.25 & -0.26 & -0.27 \\
\hline Middle UA-S/D ratio & & -0.38 & -0.40 & -0.41 & -0.40 \\
\hline Middle UA-Doppler* & & Late BPD & Late $\mathrm{HC}$ & Late AC & Late FL \\
\hline Middle UA-PI & & 0.05 & 0.01 & 0.01 & 0.01 \\
\hline Middle UA-RI & & -0.01 & 0.05 & -0.02 & -0.02 \\
\hline Middle UA-S/D ratio & & 0.00 & 0.01 & -0.05 & -0.01 \\
\hline In late pregnancy & Birth weight & Late BPD & Late $\mathrm{HC}$ & Late AC & Late FL \\
\hline Late UA-PI & $-0.24^{\star}$ & $-0.14^{\star}$ & $-0.16^{\star}$ & $-0.22 \bullet$ & $-0.15^{\star}$ \\
\hline Late UA-RI & $-0.22 \bullet$ & $-0.15^{\star}$ & $-0.14^{\star}$ & -0.18 & $-0.13^{\star}$ \\
\hline Late UA-S/D ratio & $-0.19^{\star}$ & -0.05 & -0.05 & $-0.16^{\bullet}$ & -0.08 \\
\hline \multicolumn{6}{|c|}{ in GDM group } \\
\hline In middle pregnancy & & Middle BPD & Middle HC & Middle AC & Middle FL \\
\hline Middle UA-PI & & $-0.34^{\star}$ & $-0.39^{\bullet}$ & $-0.34^{\star}$ & $-0.39^{\star}$ \\
\hline Middle UA-RI & & $-0.27^{\bullet}$ & $-0.32^{\bullet}$ & $-0.30^{\star}$ & $-0.31^{\star}$ \\
\hline Middle UA-S/D ratio & & $-0.38^{\bullet}$ & $-0.41^{\bullet}$ & $-0.37^{\bullet}$ & $-0.41^{\star}$ \\
\hline Middle UA-Doppler* & & Late BPD & Late $\mathrm{HC}$ & Late AC & Late FL \\
\hline Middle UA-PI & & -0.03 & -0.06 & 0.01 & -0.06 \\
\hline Middle UA-RI & & -0.07 & -0.06 & -0.06 & -0.07 \\
\hline Middle UA-S/D ratio & & -0.03 & -0.05 & 0.02 & -0.02 \\
\hline In late pregnancy & Birth weight & Late BPD & Late $\mathrm{HC}$ & Late AC & Late FL \\
\hline Late UA-PI & -0.09 & 0.00 & 0.01 & 0.02 & -0.03 \\
\hline Late UA-RI & -0.08 & 0.01 & 0.01 & 0.01 & 0.00 \\
\hline Late UA-S/D ratio & -0.08 & -0.02 & 0.02 & -0.01 & -0.01 \\
\hline \multicolumn{6}{|c|}{$\begin{array}{l}\text { *indices with late ultrasound parameters. UA umbilical artery, S/D systolic/diastolic } \\
\text { ratio, PI pulsatility index, RI resistance index, BPD biparietal diameter,HC head } \\
\text { circumference, AC abdominal circumference, FL femur length, Pearson's correlation } \\
\text { coefficient is given for bivariate correlation between all pairs. 'Statistically significant } \\
(\mathrm{P}<0.05) \text {. }\end{array}$} \\
\hline
\end{tabular}

ween umbilical blood flow and fetal growth in GDM and non-GDM pregnancy (for more details see table 2).

Fetal size with UA-Doppler indices by multivariable regression analysis

We subsequently analyzed the correlation by multivariable regression analysis considering gestational day of the ultrasound examination, the child's sex, maternal BMI before pregnancy, maternal age at delivery, maternal body weight at delivery and maternal hypertensive disorder as confounding factors. Both in GDM group and non-GDM group, the late pregnancy UA-Doppler indices were highly negative related to infant birth weight. Besides that, it was showed that the late UA-Doppler indices (PI, RI and S/D ratio) was negative related to birth weight, BPD, HC, AC, FL in non-GDM group, whereas the middle UA-Doppler indices (PI, RI and S/D ratio) was negative related to HC and FL in the GDM group (for more details see table 3,4 and figure 1 ).

\section{Discussion}

In the current birth cohort study, we showed that UA-Doppler indices (PI, RI and S/D ratio) were associated with fetal head circumference and femur length development in GDM middle pregnancy, whereas (PI, RI and S/D ratio) were associated with the whole fetal organ (biparietal diameter, head circumference, abdominal circumference, femur length) development in non-GDM late pregnancy only. This indicates that there were obviously different time 


\section{Kidney Blood Pressure Research}

windows during pregnancy where umbilical blood flow was critical for fetal development in GDM and non-GDM pregnancy.

The cohort studied here is representative in terms of key characteristics for a Chinese cohort like maternal age, maternal BMI before pregnancy, gestational age [12], and birth weight [13] suggesting that our findings are of general impact.

Several fetal and placental circulation Doppler derived indices such as maternal uterine arteries, umbilical artery and those of the fetal circulation have been used in clinical practice to identify fetuses who are at risk of increased perinatal morbidity that may benefit from closer surveillance or elective delivery. Among them UA-PI, RI and S/D ratio are used most commonly, because these indices are an estimate of placental impedance to blood flow. UA-PI reflects peripheral resistance. UARI reflects the vascular elasticity. UA-S/D ratio reflects the impedance of the placenta. In pa-

\section{Kidney Blood Press Res 2014;39:369-377}

DOI: 10.1159/00035581

Publisned ontIne: september 30, 2014

(C) 2014 S. Karger AG, Base

www.karger.com/kbr

i/Chen/Dong/Yu/Lu/Xiao/Hocher: Umbilical Blood Flow Regulation and Fetal Development

Table 3. Multivariable regression analysis of the association of fetal size with UA-Doppler indices in middle pregnancy

\begin{tabular}{|c|c|c|c|c|}
\hline \multicolumn{5}{|c|}{ non-GDM group } \\
\hline & Middle BPD & Middle HC & Middle AC & Middle FL \\
\hline \multicolumn{5}{|c|}{ Middle UA-PI. } \\
\hline $\mathrm{R}^{2}$ & 0.091 & 0.088 & 0.071 & 0.073 \\
\hline B & 1.439 & 2.346 & -0.164 & 0.167 \\
\hline P-Value & 0.546 & 0.467 & 0.975 & 0.855 \\
\hline \multicolumn{5}{|c|}{ Middle UA-RI. } \\
\hline $\mathrm{R}^{2}$ & 0.094 & 0.096 & 0.072 & 0.076 \\
\hline B & -1.451 & 8.345 & -9.668 & -1.671 \\
\hline P-Value & 0.258 & 0.230 & 0.401 & 0.399 \\
\hline \multicolumn{5}{|c|}{ Middle UA S/D ratio } \\
\hline $\mathrm{R}^{2}$ & 0.082 & 0.088 & 0.068 & 0.067 \\
\hline B & 0.051 & $0.550^{\bullet}$ & -2.113 & -0.129 \\
\hline P-Value & 0.869 & 0.047 & 0.168 & 0.622 \\
\hline \multicolumn{5}{|c|}{ GDM group } \\
\hline & Middle BPD & Middle HC & Middle AC & Middle FL \\
\hline \multicolumn{5}{|c|}{ Middle UA-PI. } \\
\hline $\mathrm{R}^{2}$ & 0.824 & 0.875 & 0.819 & 0.805 \\
\hline B & -0.999 & $-9.271^{\star}$ & -4.343 & $-2.814^{\star}$ \\
\hline P-Value & 0.324 & 0.004 & 0.262 & 0.002 \\
\hline \multicolumn{5}{|c|}{ Middle UA-RI. } \\
\hline $\mathrm{R}^{2}$ & 0.802 & 0.863 & 0.803 & 0.780 \\
\hline B & -1.977 & $-15.351 \bullet$ & -12.526 & $-4.209 \bullet$ \\
\hline P-Value & 0.264 & 0.005 & 0.055 & 0.007 \\
\hline \multicolumn{5}{|c|}{ Middle UA S/D ratio } \\
\hline $\mathrm{R}^{2}$ & 0.818 & 0.088 & 0.816 & 0.797 \\
\hline B & -0.343 & $0.550^{\bullet}$ & -0.788 & $-0.649^{\bullet}$ \\
\hline P-Value & 0.240 & 0.027 & 0.478 & 0.012 \\
\hline
\end{tabular}

For each ultrasound measurement, the multivariable regression analyses were performed, with the ultrasound measurement being the dependent and each of the UA-Doppler indices being the independent variable. Shown are the B and P-values for the UA-Doppler indices. B non-standardized regression coefficient. ${ }^{2}$; regression coefficient for the entire model with all confounding factors: UA umbilical artery, S/D systolic/diastolic ratio, PI pulsatility index, RI resistance index, BPD biparietal diameter, HC head circumference, AC abdominal circumference, FL femur length. The following confounding factors were included into the models: gestational day of the ultrasound examination, the child's sex, maternal BMI before pregnancy, maternal age at delivery, maternal body weight at delivery and maternal hypertension. - Statistically significant $(\mathrm{P}<0.05)$

thological pregnancy such as fetal growth restriction, these parameters indicate impairment of placental blood flow and function, and are used for monitoring high risk pregnancy [14]. In contrast to our study, Tan et al.'s study [15] showed that diabetic pregnancy was not associated with a significantly altered umbilical blood flow. However, another study by Reitte et al. [16] showed that Doppler flow examinations with RI measurements in patients with GDM differed significantly with respect to healthy controls. The reason why Tan et al. did not find differences was mostly likely due to the fact that the sample size analyzed in their study was much too small. They had just 50 GDM cases versus 50 control cases. We had 226 GDM cases versus 519 non-GDM cases. Tan et al. just analyzed ultrasound parameters in 36th gestational week but not in middle gestation.

A close linear relationship between birth weight and UA-Doppler velocity waveforms in late pregnancy has been described previously [17]. Our data are in agreement with this 


\section{Kidney Blood Pressure Research}

study. However, our study is the first to demonstrate in a large well characterized birth cohort that the impact of umbilical blood flow on fetal development is substantially different in GDM and non-GDM pregnancy. We demonstrated that in particular umbilical blood flow was critical for organ development in middle GDM pregnancy only. This is reflected by the fact that measures of the body shape in middle GDM pregnancy (day 160 of gestation) were in general smaller as compared to non-GDM pregnancy. Head circumference, biparietal diameter, abdominal circumference and femur length, all these parameters were smaller in fetus of GDM pregnancy versus non-GDM pregnancy (see table 1), whereas in late pregnancy measures of the fetal body are in general larger in GDM pregnancy. We suggest that maternal diabetes associated factors such as glycated end products might impair endothelial function of the umbilical artery in particular in mid gestation. Both
Kidney Blood Press Res 2014;39:369-377

\begin{tabular}{l|l}
\hline DOI: $10.1159 / 000355815$ & C 2014 S. Karger AG, Basel
\end{tabular}

Publisnea onIIne: September 30, 2014

www.karger.com/kbr

Li/Chen/Dong/Yu/Lu/Xiao/Hocher: Umbilical Blood Flow Regulation and Fetal Development

Table 4. Multivariable regression analysis of the association of fetal size with UA-Doppler indices in late pregnancy

\begin{tabular}{|c|c|c|c|c|c|}
\hline \multicolumn{6}{|c|}{ non-GDM group } \\
\hline & Birth weight & Late BPD & Late $\mathrm{HC}$ & Late AC & Late FL \\
\hline \multicolumn{6}{|l|}{ Late UA-PI. } \\
\hline $\mathrm{R}^{2}$ & 0.443 & 0.528 & 0.524 & 0.577 & 0.539 \\
\hline B & $-395.979^{\star}$ & -1.738 & -5.122 & $-18.917^{\bullet}$ & -.473 \\
\hline P-Value & 0.0001 & 0.051 & 0.057 & 0.001 & .068 \\
\hline \multicolumn{6}{|l|}{ Late UA-RI. } \\
\hline $\mathrm{R}^{2}$ & 0.425 & 0.529 & 0.520 & 0.565 & 0.592 \\
\hline B & $-579.547^{\bullet}$ & $-5.537^{\bullet}$ & -11.958 & $-32.481 \bullet$ & $-2.851 \bullet$ \\
\hline P-Value & 0.001 & 0.001 & 0.015 & 0.001 & 0.030 \\
\hline \multicolumn{6}{|c|}{ Late UA-S/D ratio } \\
\hline $\mathrm{R}^{2}$ & .376 & .454 & .444 & .524 & .539 \\
\hline B & $-115.719^{\bullet}$ & -.339 & -.488 & $-6.688^{\bullet}$ & -.473 \\
\hline P-Value & 0.001 & 0.290 & 0.610 & 0.001 & 0.078 \\
\hline \multicolumn{6}{|c|}{ GDM group } \\
\hline & Birth weight & Late BPD & Late $\mathrm{HC}$ & Late AC & Late FL \\
\hline \multicolumn{6}{|l|}{ Late UA-PI. } \\
\hline $\mathrm{R}^{2}$ & 0.345 & 0.507 & 0.583 & 0.609 & 0.676 \\
\hline B & $-254.757^{\bullet}$ & -.145 & -1.150 & -2.650 & -1.200 \\
\hline P-Value & 0.020 & 0.891 & 0.678 & 0.577 & 0.103 \\
\hline \multicolumn{6}{|l|}{ Late UA-RI. } \\
\hline $\mathrm{R}^{2}$ & 0.340 & 0.511 & 0.579 & 0.609 & 0.672 \\
\hline B & $-612.426^{\star}$ & 0.456 & -2.242 & -8.063 & -1.475 \\
\hline P-Value & 0.014 & 0.849 & 0.724 & 0.459 & 0.380 \\
\hline \multicolumn{6}{|c|}{ Late UA-S/D ratio } \\
\hline $\mathrm{R}^{2}$ & 0.318 & 0.522 & 0.586 & 0.609 & 0.682 \\
\hline B & -61.839 & -.199 & 1.049 & -8.063 & -.122 \\
\hline P-Value & 0.231 & 0.681 & 0.418 & 0.809 & 0.719 \\
\hline
\end{tabular}

For each ultrasound measurement, the multivariable regression analyses were performed, with the ultrasound measurement being the dependent and each of the UA-Doppler indices being the independent variable. Shown are the $\mathrm{B}$ and $\mathrm{P}$-values for the UA-Doppler indices. $\mathrm{R}^{2}$; regression coefficient $B$ non-standardized regression coefficient. $R^{2}$; regression coefficient for the entire model with all confounding factors. UA umbilical artery, S/D systolic/diastolic ratio, PI pulsatility index, RI resistance index, BPD biparietal diameter, HC head circumference, AC abdominal circumference, FL femur length. The following confounding factors were included into the models: gestational day of the ultrasound examination, the child's sex, maternal BMI before pregnancy, maternal age at delivery, maternal body weight at delivery and maternal hypertension. \$Statistically significant $(\mathrm{P}<0.05)$

the consequences for the organ function of the offspring and the underlying mechanism needs to be explored in further studies.

The frequency of GDM is rather high in our study as compared to others. There are several reasons that might explain it. First, we analyzed a southern Asian population. It is known that the incidence of GDM is much higher in this population as compared to Caucasian populations. Second, the rate of diabetes and also GDM is currently rising in China due to changes in the way of nutrition intake. Western style diet becomes more and more frequent in larger Chinese cities. Third, the definition of GDM changed. We used the modern definition. This will clearly increase the number of GDM cases. We used the following criteria according to the current guidelines: plasma glucose concentrations were measured at 0,60 , and 120 minutes when the woman received $75 \mathrm{~g}$ glucose in the second trimester of pregnancy. GDM was diagnosed when patient plasma glucose levels exceeded or reached just one of the following thresholds: 


\section{Kidney \\ Blood Pressure Research}

Kidney Blood Press Res 2014;39:369-377

\begin{tabular}{l|l}
\hline DOI: 10.1159/000355815 & (c 2014 S. Karger AG, Basel \\
\hline
\end{tabular}

Publisned onine: september 30, 2014

www.karger.com/kbr

Li/Chen/Dong/Yu/Lu/Xiao/Hocher: Umbilical Blood Flow Regulation and Fetal Development

fasting glucose $\geq 5.1 \mathrm{mmol} / \mathrm{L}$; one hour glucose $\geq 10.0 \mathrm{mmol} / \mathrm{L}$ or two hour glucose $\geq 8.5 \mathrm{mmol} / \mathrm{L}$ [5]. In former times (before 2011), we used the American Diabetes Association (ADA) GDM criteria [18], plasma glucose levels had to exceed or reached at least two times the thresholds: fasting glucose $\geq 5.3 \mathrm{mmol} / \mathrm{L}$; one hour glucose $\geq 10.3 \mathrm{mmol} / \mathrm{L}$ or two hour glucose $\geq 8.6 \mathrm{mmol} / \mathrm{L}$. These three facts together might explain the rather high frequency of GDM in our population. In essence, we have more, but in average milder forms of GDM pregnancy in our cohort due to the change in the diagnose criteria as described above. Beside the improved treatment of GDM nowadays in Chinese university hospitals (with treatment after diagnosis in the 2nd trimester, most ladies get pretty normal HbA1C values), the alteration in the diagnostic criteria might also explain why birth weight and other anthropomorphic measures of newborns size at birth were just numerically but not statistically higher in GDM pregnancy.

\section{Conclusion}

We showed that UA-Doppler indices (PI, RI and S/D ratio) were associated with fetal head circumference and femur length in middle GDM pregnancy. We showed for the first time that head circumference, biparietal diameter, abdominal circumference and

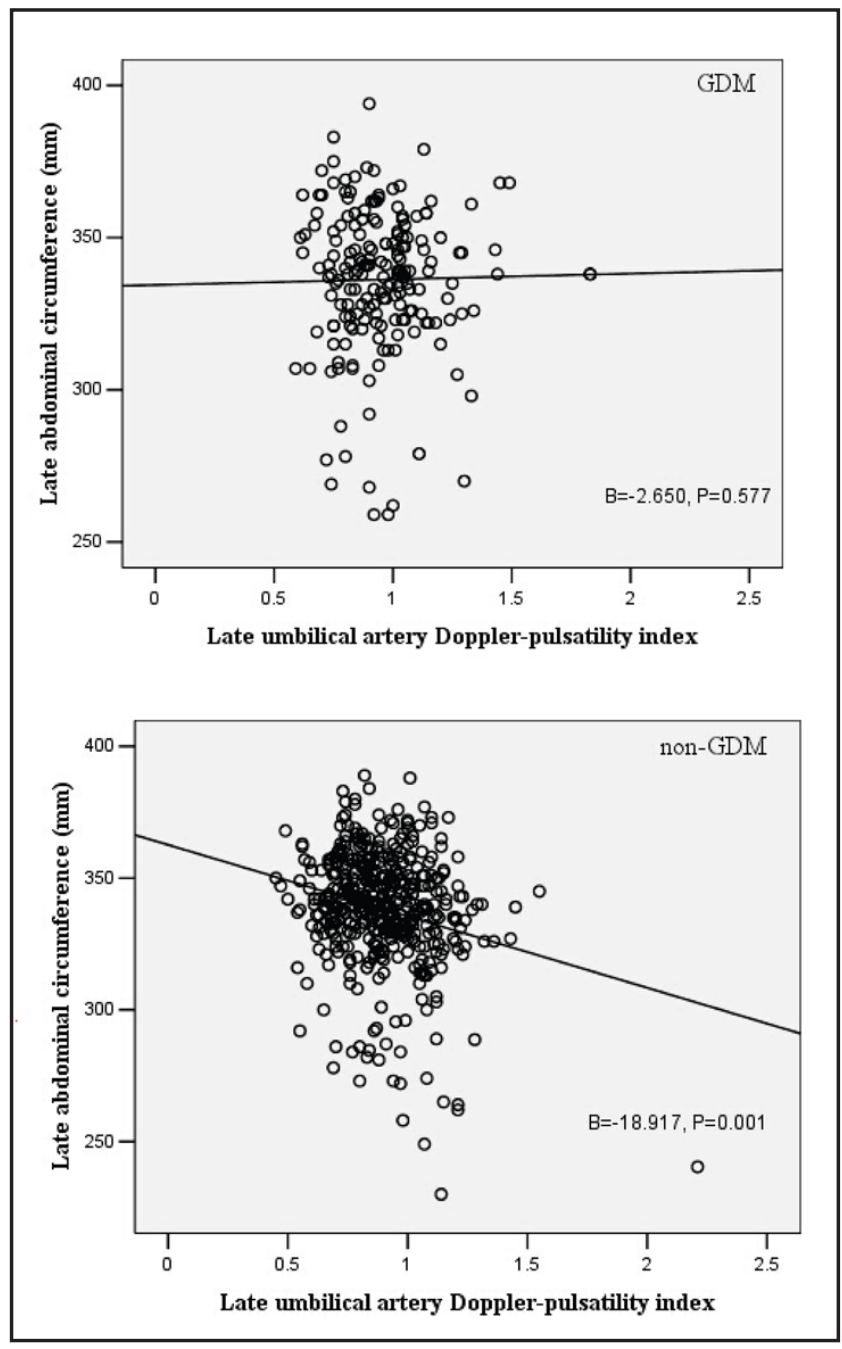

Fig. 1. Relationship between late umbilical artery Doppler-pulsatility index and late abdominal circumference in GDMand non-GDM-pregnancies. For statistical analysis of the association, we performed a multivariable regression analysis, see table 4 . The following confounding factors were included into the models: gestational day of the ultrasound examination, the child's sex, maternal BMI before pregnancy, maternal age at delivery, maternal body weight at delivery and maternal hypertension. femur length in middle gestation were smaller in fetus of GDM pregnancy versus non-GDM pregnancy. The physiological and molecular pathways why there is a catch up growth in later times of GDM pregnancy resulting in larger GDM babies at birth needs to be addressed in further studies.

\section{Disclosure Statement}

The authors declared no conflicting interests. 


\section{Kidney \\ Blood Pressure Research}

\section{Acknowledgment}

This study was partially supported by a research grant from Hoffmann La Roche, Basel, Switzerland and the Chinese National Natural Science Foundation of China (no. 81300557). The authors gratefully acknowledge the contributions of the general practitioners and midwives.

\section{References}

1 Rathmann W, Giani G: Global prevalence of diabetes: Estimates for the year 2000 and projections for 2030. Diab Care 2004;27:2568-2569.

- Yang SH, Dou KF, Song WJ: Prevalence of diabetes among men and women in china. N Engl J Med 2010;362:2425-2426.

-3 Mocarski M, Savitz DA: Ethnic differences in the association between gestational diabetes and pregnancy outcome. Matern Child Health J 2012;16:364-373.

4 Thorpe LE, Berger D, Ellis JA, Bettegowda VR, Brown G, Matte T, Bassett M, Frieden TR: Trends and racial/ ethnic disparities in gestational diabetes among pregnant women in new york city, 1990-2001. Am J Publ Health 2005;95:1536-1539.

-5 Coustan DR:Diagnosis of gestational diabetes. Scan J Clin Lab Invest 2014;74:27-33.

6 Dabelea D, Snell-Bergeon JK, Hartsfield CL, Bischoff KJ, Hamman RF, McDuffie RS: Increasing prevalence of gestational diabetes mellitus (gdm) over time and by birth cohort: Kaiser permanente of colorado gdm screening program. Diab Care 2005;28:579-584.

7 Boney CM, Verma A, Tucker R, Vohr BR: Metabolic syndrome in childhood: Association with birth weight, maternal obesity, and gestational diabetes mellitus. Pediatrics 2005;115:e290-296.

8 Bunt JC, Tataranni PA, Salbe AD: Intrauterine exposure to diabetes is a determinant of hemoglobin a(1)c and systolic blood pressure in pima indian children. J Clin Endocrinol Metab 2005;90:3225-3229.

-9 Ishida K, Taguchi K, Matsumoto T, Kobayashi T: Activated platelets from diabetic rats cause endothelial dysfunction by decreasing akt/endothelial no synthase signaling pathway. PloS one 2014;9:e102310.

10 Celik A, Topuz M, Gozukara Y, Gundes A, Yesil E, Ovla D, Ozcan IT: the relationship between epicardial adipose tissue and endothelial dysfunction in patients with type 2 diabetes mellitus. Turk Kardiyoloji Dernegi arsivi 2014;42:450-455.

11 Li J, Wang ZN, Chen YP, Dong YP, Shuai HL, Xiao XM, Reichetzeder C, Hocher B: Late gestational maternal serum cortisol is inversely associated with fetal brain growth. Neurosci Biobehav Rev 2012;36:1085-1092.

12 Liu Z, Ao D, Yang H, Wang Y: Gestational weight gain and risk of gestational diabetes mellitus among chinese women. Chin Med J 2014;127:1255-1260.

13 He Y, Wen S, Tan H, Zhou S, Guo Y, Wang S, Shen L, Liu Y, Hu L: study on the influence of pregnancy-induced hypertension on neonatal birth weight and its interaction with other factors. Zhonghua liu xing bing xue za zhi 2014;35:397-400.

14 McCowan LM, Mullen BM, Ritchie K: Umbilical artery flow velocity waveforms and the placental vascular bed. Am J Obstet Gynecol 1987;157:900-902.

15 Tan AE, Norizah WM, Rahman HA, Aziz BA, Cheah FC: Umbilical artery resistance index in diabetic pregnancies: The associations with fetal outcome and neonatal septal hypertrophic cardiomyopathy. J Obstet Gynaecol Res 2005;31:296-301.

16 Reitter A, Hajduk B, Geka F, Buxmann H, Schlosser R, Louwen F: Doppler studies of gestational diabetes in the third trimester. Ultraschall Med 2011;32:E162-E168.

17 Bonnin P, Bailliart O, Kedra W, Ciraru-Vigneron N, Niang E, Savin E, Martineaud JP: Relationship between birth weight and umbilical doppler blood flow velocity waveforms during the third trimester of pregnancy. Eur J Med 1993;2:219-222.

18 Agarwal MM, Dhatt GS, Zayed R, Bali N: Gestational diabetes: relevance of diagnostic criteria and preventive strategies for Type 2 diabetes mellitus. Arch Gynecol Obstet 2007;276:237-243. 